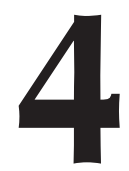

\title{
MEDIDAS PARA COMBATIR EL PLAGIO EN LOS PROCESOS DE APRENDIZAJE
}

\section{(MEASURES TO COMBAT PLAGIARISM IN LEARNING PROCESSES)}

Jesús Miguel Muñoz Cantero

Eva María Espiñeira Bellón

María Cristina Pérez Crego

Universidade da Coruña

DOI: $10.5944 / e d u c X X 1.28341$

Cómo referenciar este artículo/How to reference this article:

Muñoz Cantero, J.M., Espiñeira Bellón, E.M., y Pérez Crego, M.C., (2021). Medidas para combatir el plagio en los procesos de aprendizaje. Educación XX1, 24(2), 97-120. https://doi.org/10.5944/ educXX1.28341

Muñoz Cantero, J.M., Espiñeira Bellón, E.M., \& Pérez Crego, M.C., (2021). Measures to combat plagiarism in learning processes. Educación XX1, 24(2), 97-120. https://doi.org/10.5944/ educXX1.28341

\section{RESUMEN}

Este estudio se centra en determinar la percepción del alumnado universitario sobre posibles estrategias de eliminación del plagio en sus trabajos académicos. Se emplea una metodología cualitativa, de corte interpretativo e inductivo a partir de la respuesta a una pregunta abierta orientada a determinar cómo se puede evitar el plagio, incluida en el Cuestionario de Atribuciones para la Detección de Coincidencias en Trabajos Académicos (CUDECO). Las categorías que surgen del discurso de las personas encuestadas se centran en: (a) información; normalmente con respecto al proceso de citación, (b) formación; tanto del proceso de citación como de la elaboración de los trabajos que se solicitan (c) supervisión; por parte del profesorado y (d) medidas sancionadoras; coercitivas y punitivas. Las principales formas de combatir el plagio según el estudiantado, se centran en la propia definición de lo que ha de entenderse por 
plagio académico y, en medidas informativas (normalmente con respecto al proceso de citación), formativas (tanto por parte del profesorado como de las facultades y la propia institución), de carácter digital (a través de herramientas de detección), de supervisión por parte del profesorado (concienciando en la importancia de citar correctamente, produciéndose una mayor implicación y un mayor interés tanto por el tipo de trabajos que se solicitan y los tiempos asignados como por el carácter de las evaluaciones que se realizan) pero también coercitivas y punitivas. Existen algunos casos en los que se valora la necesidad de comenzar en dicho proceso con anterioridad, en cursos anteriores a los universitarios y también casos que consideran que es algo inevitable de difícil solución. En conclusión, reflexionar en torno a estas categorías, intentando combatirlas, podría disminuir el plagio en las universidades.

\section{PALABRAS CLAVE}

Plagio, enseñanza superior, ciencias sociales, estudiante universitario, estrategias educativas

\section{ABSTRACT}

This study focuses on determining the perception of university students about possible strategies to eliminate plagiarism from their academic work. A qualitative, interpretative and inductive methodology based on the answer to an open question aimed at determining how plagiarism can be avoided, included in the Attributes Questionnaire for the Detection of Coincidences in Academic Work (CUDECO). The categories that emerge from the discourse of the respondents focus on: (a) information; normally regarding the citation process, (b) training; both the citation process and the preparation of the works requested (c) supervision; by the teaching staff and (d) sanctioning measures; both coercive and punitive. The main ways to combat plagiarism, according to the student body, focus on the definition of what is to be understood by academic plagiarism and on informative measures (usually with respect to the citation process), formative measures (both by the teachers and the faculty members and the institution itself), of a digital nature (through detection tools), of supervision by the teaching staff (raising awareness of the importance of quoting correctly, producing greater involvement and greater interest in both the type of work that is requested and the times assigned as per the nature of the evaluations that are carried out) but also coercive and punitive. There are some cases where there is a need to start with this process beforehand, in courses prior to university studies, and also cases considered to be somewhat inevitable, difficult to solve. In conclusion, reflecting on these categories and trying to combat them could reduce plagiarism in universities. 


\section{KEYWORDS}

Plagiarism, higher education, social sciences, university students, educational strategies

\section{INTRODUCCIÓN}

La elaboración de trabajos académicos constituye una de las tareas más frecuentes del alumnado universitario, especialmente de la rama de Ciencias Sociales y Jurídicas, a través de los cuales se pone en juego la capacidad de reflexión y cuestionamiento crítico, basándose en la fundamentación teórica a la cual se accede a través del proceso de enseñanza y aprendizaje establecido por el profesorado, por otras fuentes documentales, o a través de las TIC como estilo pedagógico (Cordero, citado en Caldevilla, 2010; Mendoza, 2019). De esta forma, Ponte (2020) resalta la importancia de una buena adquisición de competencias científicas en el estudiantado universitario ya que en este nivel tiene, en la mayoría de las ocasiones, que desarrollar trabajos de investigación para lograr una titulación académica.

Este proceso se ve influenciado, en ocasiones, por la facilidad de obtención de información sin reconocimiento de la autoría y por ello, numerosos estudios se centran en recoger datos sobre la utilización de la fórmula de "copiar y pegar" (Dias et al., 2013; Mejía y Ordóñez, 2004; García-Peñalvo, 2017) e insisten en reconocer que el alumnado copia, parcial o totalmente, y solo una pequeña parte cita correctamente (Caldevilla, 2010) así como que esta situación no comienza en el ámbito universitario sino que el estudiantado llega a la universidad con dicha práctica "convertida en hábito" (Miranda, 2013; Morey-López et al., 2013).

Dado que esta práctica parece ser cada vez más generalizada, las universidades han de tomar conciencia sobre ello con el fin de remediarlo y determinar cuáles podrían ser las medidas para prevenirla (Cebrián-Robles et al., 2016; Sarmiento-Campos et al., 2019). En este sentido, este estudio plantea, como objetivo principal, investigar cómo se puede evitar el plagio (copiar sin citar) partiendo de la percepción de estudiantes de grado y máster de la rama de Ciencias Sociales y Jurídicas de la Universidade da Coruña (Proyecto de investigación financiado por la Xunta de Galicia -España- con referencia 002/2019).

\section{MARCO TEÓRICO}

En el ámbito académico, debe primar la originalidad de los escritos, tal y como se establece en los principios éticos y morales de las normas universitarias, respetando el trabajo de autorías precedentes, ya que el crecimiento intelectual de una persona se basa en el análisis e interpretación de estudios precedentes 
y no en la copia de ideas ajenas. Esta manera de aprender hace a las personas más competentes, autónomas, valiosas,..., mientras que plagiar perjudica la formación universitaria, de tal forma que hace a las personas menos capaces, creativas e innovadoras y además puede dar lugar a responsabilidades penales y sanciones académicas.

Numerosos estudios se han centrado en abordar esta temática tratando de identificar las causas de que se produzca esta situación, tanto a nivel internacional (Kuntz y Buttler, 2014; Tayan, 2017; Zarfsaz y Ahmadi, 2017) como nacional (Cebrián-Robles et al., 2016; Cebrián-Robles et al., 2018; Froment et al., 2020) pero existen muchos menos estudios centrados en determinar las medidas para combatir el plagio académico.

Entre otros aspectos, se pueden señalar a nivel teórico, medidas centradas en la información, en la formación, en la supervisión continua a través de plataformas diseñadas para tal fin, en las sanciones, etc., como se expone a continuación.

Como consejo general, se establece que las informaciones de las universidades deben ir destinadas a señalar que no es necesario cometer plagio "ya que se puede aprender a leer y citar correctamente" (Ochoa y Cueva, 2016, p. 37). Cebrián-Robles et al. (2018) y Ramírez (2017) aconsejan además acciones referidas a cambios que a través de comités institucionales, como comités de ética, ayuden a promover el sentido de la ética en los trabajos académicos.

Se señala, por otra parte, la importante labor formativa que tiene el profesorado en diferentes momentos del proceso de enseñanza-aprendizaje, sobre todo en las actividades relacionadas con el empleo de información científica (al diseñar las asignaturas, en las tutorías y en la supervisión de los trabajos), conversando con el alumnado sobre plagio, proporcionándole habilidades en el proceso de citación o dándole feedback durante el proceso de aprendizaje (Cebrián-Robles et al., 2018; Froment et al., 2020; Rodríguez et al., 2020; Zarfsaz y Ahmadi, 2017). Se indica además que el profesorado ha de adoptar una postura pedagógica, de tal forma que, en las primeras clases, se ha de esforzar en explicar los procesos de citación y sus implicaciones, diseñar y acotar adecuadamente los temas a trabajar, y, durante el proceso, realizar un seguimiento continuo adecuándose al tiempo de que dispone para valorar el proceso de escritura del estudiantado (Ordóñez et al., 2006; Ochoa y Cueva, 2016). Las técnicas de trabajo intelectual, la búsqueda y el análisis bibliográficos destacan en la elaboración de trabajos académicos y, por ello, las habilidades de información se convierten en una necesidad del estudiantado (Rodríguez et al., 2020) como se recoge en el artículo 6 del Real Decreto 1027/2011, de 15 de julio, por el que se establece el Marco Español de Cualificaciones para la Educación Superior.

Para ello, Mejía y Ordóñez (2004) indican que es necesario efectuar un estudio acerca de las metodologías de enseñanza y de los resultados de 
aprendizaje asociados a la evaluación; concretamente, en el proceso de evaluación se recomienda incorporar criterios que lleven a reducir las calificaciones de los trabajos que se hayan plagiado (Cebrián-Robles et al., 2018).

Existe diversidad de opinión en torno a las medidas a poner en marcha en el momento en el que se detecte plagio; Miranda (2013) indica al respecto que para combatirlo es fundamental que la persona que plagia reciba la correspondiente sanción; Ordóñez et al. (2006), indican que probablemente no sea suficiente; y, en sentido contrario, Cebrián-Robles et al. (2018) señalan que no son exitosas. También se aprecian valoraciones contrapuestas en lo que respecta a los programas digitales empleados para detectar el plagio; así, Miranda (2013) y Zarfsaz y Ahmadi (2017) son partidarios/as de su utilización e indican que cuando el estudiantado conoce la existencia de estas herramientas informáticas, sí resultan preventivas; Cebrián-Robles et al. (2018) consideran, en cambio, que su empleo no es suficiente.

Finalmente, teniendo en cuenta consideraciones éticas, Miranda (2013) indica que es necesario enseñar que "el plagio es ilícito y por qué lo es" (p.723). En este sentido, en opinión de Cebrián-Robles et al. (2016) las instituciones deben fomentar una cultura ética transversalmente en las diferentes asignaturas y durante la vida académica del estudiantado. De esta forma, Escalante e Ibarra (2013) indican que el estudiantado debe cuidar que las normas éticas estén siempre presentes en los trabajos que realiza.

Dentro del proceso de enseñanza-aprendizaje, también es necesario tener en cuenta factores personales que afectan a quienes forman parte del proceso (profesorado y alumnado) indicándose, entre ellos, el cuidado/descuidado de las normas éticas, el interés/desinterés, la experiencia/inexperiencia, la permisividad/ rectitud, la seguridad/inseguridad y el conocimiento/desconocimiento (Ordóñez et al., 2006).

El valor añadido de este estudio se centra en identificar más medidas de las que ya han sido identificadas en estudios previos, así como en poder establecer un proceso de actuación docente en el que se integren dichas medidas y no se pongan en marcha de manera aislada. La diferencia con respecto a estudios similares se centra sobre todo en el tipo de metodología empleada, de tal manera que el alumnado da respuesta a una pregunta abierta y no marcada por unas respuestas preestablecidas, con lo cual, dentro del tipo de metodología cualitativa, la información será de carácter más inédito y con una mayor profundidad.

\section{METODOLOGÍA}

Se describen y analizan cualitativamente las acciones de evitación de plagio señaladas por el alumnado de grado y máster de la rama de Ciencias Sociales y Jurídicas de la Universidade da Coruña $(\mathrm{N}=1985)$ al que se le ha administrado 
el Cuestionario de Atribuciones para la Detección de Coincidencias en Trabajos Académicos (CUDECO) (Muñoz-Cantero et al., 2019). El proceso de muestreo seguido ha sido no probabilístico, intencional y por conveniencia (Akbulut et al., 2008; Kuntz y Buttler, 2014; Tayan, 2017).

El cuestionario se ha administrado en formato papel en las aulas y consta de 47 preguntas centradas en determinar las actuaciones contra el plagio efectuadas por el profesorado universitario, sobre la utilidad de citar correctamente, las acciones de plagio efectuadas por el alumnado universitario y sus compañeros/ as y las causas que les motivan a plagiar. Estas preguntas han de ser contestadas por el alumnado de acuerdo con una escala de siete opciones de respuesta desde su total desacuerdo a su total acuerdo con respecto a las afirmaciones recogidas. A continuación, figura una pregunta de carácter abierto y voluntaria, ¿cómo crees que se puede evitar el plagio (copiar sin citar)?, obteniéndose un total de 759 registros cualitativos, seguramente con un menor nivel de participación debido al tipo de respuesta (de desarrollo) en contraposición con las anteriores donde el alumnado solo tenía que marcar una opción. Esta pregunta se incluye al final del cuestionario con el objetivo de que el alumnado se pueda centrar en la temática de la investigación, tanto en relación con la información solicitada en las preguntas previas, como con la información nueva que la persona participante quiera aportar.

Con respecto al estudiantado participante, han contestado la pregunta más mujeres $(67.98 \%)$ que hombres $(31.23 \%)$, perteneciendo, en su mayoría a estudios de grado $(87.48 \%)$.

Teniendo en cuenta el desglose de titulaciones, son mayoritarias las relacionadas con la Educación (un 38.08\% de Grado y un 7.77\% de Máster), seguidas por las titulaciones relacionadas con el Derecho, las Empresas y el Turismo (un $18.31 \%$ de Grado y un $1.45 \%$ de Máster). En un porcentaje inferior, aunque muy similar entre ellas, se encuentran las titulaciones de Deporte (en un $15.41 \%$ ) y las relacionadas con el ámbito laboral (un $11.33 \%$ de Grado y un $2.63 \%$ de Máster). Finalmente, el menor número de respuestas se ha obtenido en el Grado en Gestión Industrial de la Moda (2.63\%) y el Grado en Sociología (2.37\%).

El alumnado pertenece mayoritariamente a los primeros cursos de sus correspondientes titulaciones y sus edades están comprendidas entre 18 y 24 años $(\mathrm{M}=21.44 ; \mathrm{DT}=1.002)$.

El análisis de la información ha sido realizado a través de la metodología cualitativa para comprender las perspectivas y experiencias que dicho alumnado tiene sobre las posibilidades de evitar el plagio en las aulas de Educación Superior. Esta decisión permite dar voz al alumnado y con ello conocer de primera mano las opiniones sobre el tema objeto de estudio, así como las necesidades e inquietudes que los/las discentes presentan al respecto.

Los pasos a seguir fueron los siguientes: en primer lugar, la recogida de datos; en segundo lugar, la transcripción de la información; en tercer lugar, a 
partir de las transcripciones, se realizó un análisis de la información mediante una codificación descriptiva (Miles y Huberman, 1994; Miles et al., 2009) que consistió en identificar con una o dos palabras el tema central de un párrafo o grupo de palabras que describen las claves de cada cuestión. Así, se han otorgado etiquetas a los temas emergentes con el fin de favorecer la posterior recuperación de la cita o fragmento etiquetado.

A partir del proceso de codificación, los temas fueron emergiendo de manera recurrente siendo vinculados entre sí en un proceso de sistematización. A medida que los datos se fueron analizando, se elaboró la estructura de las diferentes categorías y los códigos que dan soporte a cada una de ellas para reconstruir las categorías específicas que articulan el tema de estudio y profundizar en las percepciones que el alumnado participante emplea en la comprensión de sus propias experiencias en el aula con respecto al plagio académico.

De la misma manera que se satura la teoría que está desarrollando, se ha producido la saturación de las categorías emergentes, debido a la integración y la densidad de la teoría (al analizarse y explicar el mayor número de variaciones dentro de la teoría y cuando la relación entre las categorías emergentes obedecía al esquema lógico-explicativo del problema de investigación) y, por otra parte, con respecto a los datos, al no tener acceso a otros datos que contribuyeran al desarrollo de la investigación (Glaser y Strauss, citado en Ardila y Rueda, 2013).

Como soporte tecnológico, en la codificación de las respuestas facilitadas, se ha utilizado el programa ATLAS.ti 8, decisión tomada porque permite realizar la codificación de la información con una mayor rigurosidad y fiabilidad, mediante la asignación de etiquetas a fragmentos de texto, permitiendo así la búsqueda de patrones para establecer clasificaciones (Hwang, 2007). A partir del proceso de codificación de los datos, las categorías y códigos obtenidos en el análisis se presentan en las diferentes figuras aportadas en el estudio.

Los criterios de credibilidad con los que se ha desarrollado la investigación son los propios de la investigación cualitativa (Flick, 2014).

La fiabilidad del estudio se apoya a través del tratamiento de la información mediante un proceso claro (Flick, 2012). El proceso de transcripción de la información se ha llevado a cabo con rigurosidad manteniendo el criterio de literalidad estilística (Pujadas, 2000). Igualmente, el registro de los datos se ha realizado a través de la asignación de un código a cada informante, respetando en todo momento la confidencialidad del alumnado participante. Además, se ha mantenido la diferenciación entre las interpretaciones del equipo investigador y las declaraciones de las personas informantes, las cuales se presentan entrecomilladas, indicando el origen de los datos a través del código identificador de cada participante. Así mismo se presenta una explicación del proceso de codificación de la información. 
La validez reposa en dos criterios de rigor utilizados en la investigación cualitativa (Creswell, 1998): por un lado, en la autocrítica reflexiva realizada sobre los sesgos que pueden afectar al proceso de investigación y sus resultados (Sandín, 2000) y por otro, teniendo en cuenta la validez comunicativa del alumnado participante (Flick, 2012). Ambos aspectos fomentan la claridad y el rigor de los estudios (Barry et al., 1999).

Con respecto a la ética del proceso de investigación, se ha entregado un documento de consentimiento para la participación en la investigación a la dirección de los centros universitarios. Previamente a la aplicación de los cuestionarios, se ha procedido a dar lectura a un documento informativo a las personas participantes. En dicho documento se establece que los resultados serán enviados a publicaciones científicas para su difusión, pero no se transmitirá ningún dato que pueda llevar a la identificación de las personas participantes, garantizando así la confidencialidad de sus datos. Esta ha sido una faceta clave durante todo el proceso metodológico. Con el fin de respetar la dignidad e integridad de las personas participantes y para salvaguardar la confidencialidad de los/las informantes, se ha realizado una codificación de la información identificativa, además de realizar un tratamiento riguroso y respetuoso de los datos obtenidos.

\section{RESULTADOS}

Los datos revelan que las principales formas para combatir el plagio en los procesos de aprendizaje del alumnado no se muestran como medidas aisladas, sino que forman parte de un proceso de actuación docente con cuatro fases interrelacionadas.

La primera fase comienza en el momento en el que el alumnado accede a la universidad. Este entra en las aulas con una necesidad latente de recibir información sobre las actuaciones que se consideran correctas para la adquisición de unos buenos hábitos en el desarrollo de trabajos académicos, dada la poca o nula experiencia previa que trae en su haber sobre estas temáticas. Una vez informado, es el momento de articular las medidas formativas correspondientes al proceso de citación y de elaboración de trabajos que funcionarán como soporte del aprendizaje, pasando así a la segunda fase. Para poder comprobar que este aprendizaje ha dejado huella en el alumnado, se avanzaría a la tercera fase de supervisión. En el caso del alumnado que al superar la fase tres, muestre indicios de acciones fraudulentas, pasaría a una última fase de medidas sancionadoras acordes a la infracción cometida.

A partir del proceso de codificación de los datos, las categorías principales obtenidas en el análisis se muestran en la figura 1. 


\section{Figura 1}

Categorías y códigos de análisis. Los rectángulos denotan las categorías y códigos de análisis. Los conectores entre los rectángulos responden a la siguiente leyenda: $N=e$ s causa de, $G=e s$ parte de, $O=e s$ un y $R=e s t a ́$ asociado con

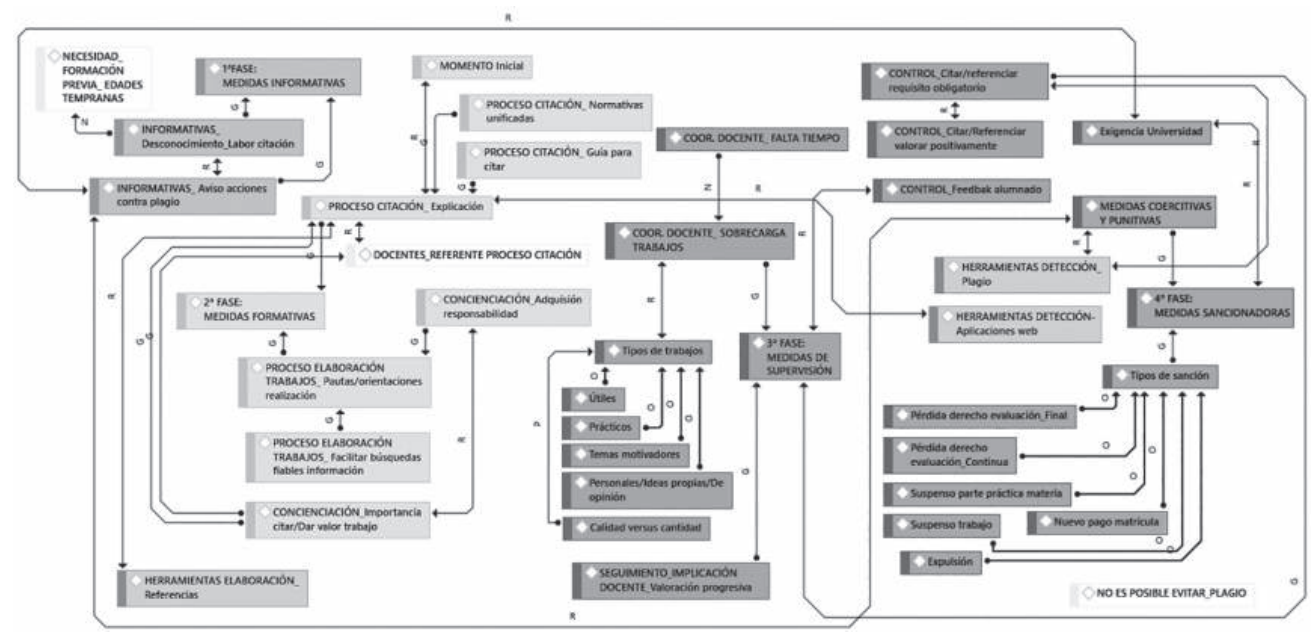

A continuación, se presentan y desarrollan las evidencias que avalan cada fase del proceso.

\section{Primera fase: medidas informativas}

Los testimonios, de acuerdo con lo reflejado en la figura 2, indican que cuando el alumnado accede a la universidad, lo hace con desconocimiento del proceso de citación, y además, una parte de este considera que estas acciones vienen arraigadas desde las etapas educativas anteriores; indicando lo siguiente: "Creo que se puede evitar informando bien a los alumnos de cómo citar correctamente. Cuando citamos mal o no citamos creo que es por desconocimiento en gran parte" (I12, 1:12) o

Pienso que una buena solución sería dar formación a los alumnos desde que somos pequeños (Primaria, ESO, Bachiller) porque muchos de nosotros llegamos a la universidad sin saber citar y nos vemos obligados a aprender casi por nuestra propia cuenta de un día para otro. El formar a los alumnos a citar las fuentes usadas desde que empezamos a hacer trabajos más serios yo creo que sería lo mejor opción (I137, 1:141).

Teniendo en cuenta lo anteriormente expuesto, un primer paso ha de centrarse en proporcionar información sobre el significado de plagio, así como de las consecuencias que tales actuaciones implican al discente "Informando al 
alumnado sobre el plagio. Dando más sesiones informativas sobre qué es y por qué es tan relevante no cometer plagio" (I398, 1:404) o

El plagio se puede evitar enseñando a citar desde el primer día, así como la manera de acceder a fuentes de información y recursos ofrecidos por las universidades, no dando por hecho que ya se sabe. Además, informando de las sanciones y la normativa universitaria referente al tema, y fundamentalmente motivando a los profesores para que se impliquen en la causa, siempre desde un punto de vista que favorezca y ayude al alumnado (I482, 1:484).

Con respecto a las conceptualizaciones sobre el plagio y lo que esto significa, se aprecian opiniones dispares como:

No me parece importante el plagio en casos de trabajos que no implican una remuneración económica o una repercusión mediática, como es el caso de los trabajos que hacemos en clase. Personalmente, no me gusta leer un trabajo que sea todo citar, aunque le añada valor científico (I84, 1:87).

Se puede evitar el plagio, primero dando crédito a todo el material utilizado y con varios procedimientos. Tomar siempre los datos de la fuente de la cual obtenemos la información. Citar la frase o el parafraseo directamente del original acreditando la autoría del trabajo. Parafrasear la autoría del trabajo. Parafrasear las palabras utilizadas por el autor entre comillas (I566, 1:565).

Figura 2

Categoría y códigos de medidas informativas. Los rectángulos denotan las categorías y códigos de análisis. Cada rectángulo recoge información que responde a la siguiente leyenda: $E=$ frecuencia y $D=$ densidad (número de códigos con los que está vinculado)

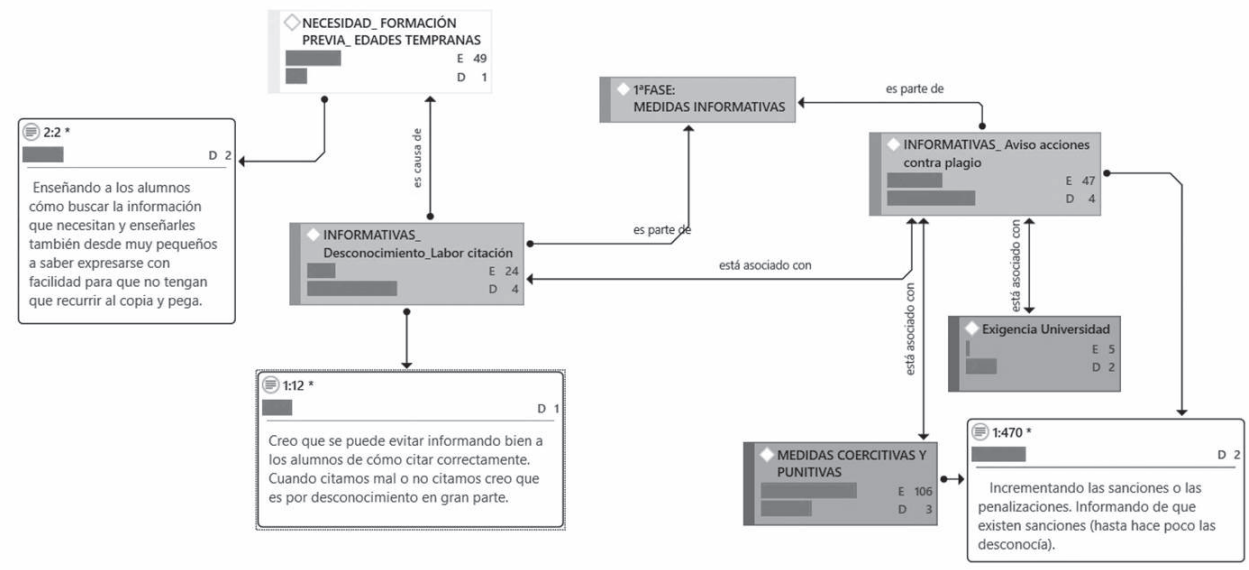




\section{Segunda fase: medidas formativas}

La segunda fase, como se aprecia en la figura 3, aborda medidas formativas, a través de las cuales el alumnado hace hincapié en la necesidad de recibir formación en dos procesos de enseñanza: en el proceso de citación de fuentes y en el de la propia elaboración de los trabajos académicos.

Con respecto al proceso de citación de fuentes bibliográficas, una muestra elevada de participantes, considera que una de las claves para evitar el plagio es una buena formación en citación "Informando de manera extensa y adecuada sobre el procedimiento que habría que seguir a la hora de recurrir a la información de terceros sin recurrir al plagio, en el momento de elaboración de los trabajos" (I546,1:546) y "Dando correctas indicaciones sobre cómo citar y ofreciendo un buen rango de información donde basarse para hacer los trabajo, que tengan las indicaciones claras y concisas para no tener problemas a la hora de citar" (I59, 1:59), reclamando normativas unificadas, donde las bases de citación presenten un consenso:

Creo que entregarle el manual (resumido) de las normas APA a los/as alumnos/as ayudaría. También creo que los/as profesores/as han de centrarse también en estas normas a la hora de que sus alumnos/as pidan una revisión antes de la corrección final de un trabajo. Se podría impartir clases sobre las normas APA con prácticas incluidas (I353, 1:353).

Se considera que el profesorado, a través de sus prácticas docentes, supone un importante referente del proceso de citación:

Primero: empezando por que los apuntes de los profesores incluyan las referencias bibliográficas. Segundo: facilitando y haciendo conocer a los alumnos la obligación de citar autores y cómo hacerlo. Tercero: en cada trabajo dedicar unos minutos cada profesor a dar una pequeña guía de cómo hacerlo (I194, 1:203).

Y finalmente, otra de las ideas recurrentes que se muestra es la necesidad de disponer de formación en citación desde el momento inicial de ingreso académico "Enseñando la normativa desde el primer momento que se entra en la carrera, y darle la importancia que tiene, es decir, que se penalice el hecho de no citar adecuadamente y se tutoricen de manera individual y grupal los trabajos" (I295, 1:298).

Con el fin de que este aspecto sea mejorable, se proponen múltiples formatos; bien a través de las propias clases, talleres o asignaturas específicas: "Sería favorable hacer un taller sobre las normas, y formas de citar, tanto el primer año de carrera como en el segundo año" (I316, 1:321), "Creo que debería existir más información sobre cómo citar, incluso alguna asignatura específica para poder practicar esto" (I205,1:214), "Como manera de evitar el plagio quizás se podría impartir una masterclass en profundidad 
explicando correctamente cómo citar e insistiendo en la importancia de hacerlo bien desde el primer minuto"(I233,1:238).

Con respecto al proceso de elaboración de los trabajos académicos, se manifiesta la necesidad de recibir pautas en la elaboración de trabajos académicos y en la búsqueda de información "Creo que la mejor manera para evitar el plagio es enseñando a los alumnos a buscar sus propias fuentes, a investigar y utilizar la bibliografía" (I608, 1:608) o

Cuando nos encargan un trabajo para clase, nos dan unas pautas mínimas para seguir, pero realmente la información que utilizamos corre de la cuenta de los y las estudiantes, a nuestro libre albedrío, provocando quizás que no sepamos en qué dirección ir ni cómo orientarnos (I273, 1:277).

Resulta imprescindible además fomentar el interés por realizar trabajos, la responsabilidad y la conciencia académica "Concienciado a la gente de que no sirve de nada copiar trabajos ya que no se aprende, todos podemos realizar un trabajo por nosotros mismos" (I322, 1:325), "Educando en conciencia académica, en la importancia de la cita en todos sus ámbitos: eleva nivel trabajo, aporta conocimiento al lector, etc.” (I610, 1:613), “Organizar una formación específica y concienciar sobre la importancia de la responsabilidad y el rigor con el trabajo académico" (I659, 1:662).

\section{Figura 3}

Categoría y códigos de medidas formativas. Los rectángulos denotan las categorías y códigos de análisis. Cada rectángulo recoge información que responde a la siguiente leyenda: E=frecuencia y $D=$ densidad (número de códigos con los que está vinculado)

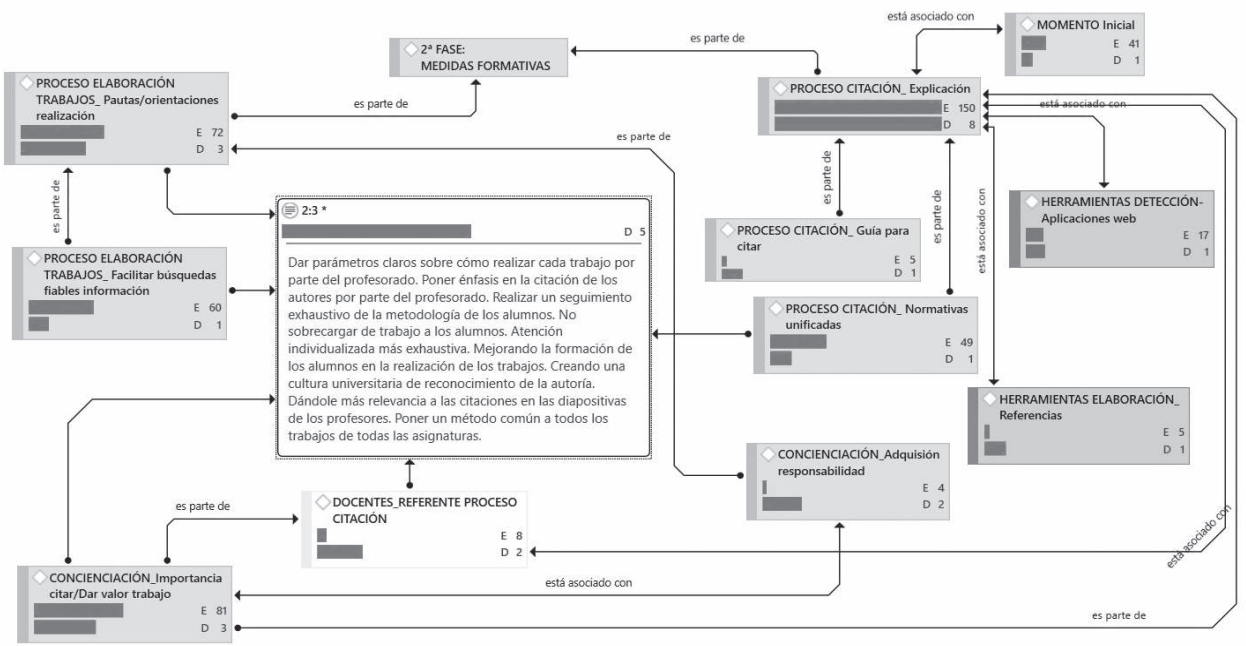




\section{Tercera fase: medidas de supervisión}

Una vez que el alumnado ha adquirido formación en el proceso de citación y ha recibido las pautas para la elaboración de trabajos, el siguiente paso supone la supervisión de la adquisición de los conocimientos y competencias adquiridos, así como las habilidades para aplicarlos (ver figura 4). De ello van a depender tres niveles de actuación: un primer nivel de coordinación docente, un segundo nivel de seguimiento y un tercer nivel de control.

Así, en primer lugar, el alumnado se refiere a la categoría de coordinación docente, manifestando que del personal docente va a depender que exista un equilibrio entre la carga de trabajo, adecuándose al tiempo de que disponen los alumnos para la realización de las tareas "El plagio podría evitarse dando la información y el tiempo necesario al alumnado para que verdaderamente pueda investigar sobre el tema del trabajo, teniendo en cuenta que tenemos más asignaturas y, en consecuencia, más trabajos" (I349, 1:349), "El plagio puede evitarse si lo que se le exige al alumnado se adapta a su tiempo y conocimiento" (I218, 1:224), "Coordinándose con otros docentes y adecuando la carga de trabajos al tiempo disponible por cada asignatura, además de facilitar el acceso al alumnado a las fuentes de información" (I10,1: 10), "Quizás sea mejor hacer un mismo trabajo que incluya dos asignaturas, de forma que el alumnado disponga de más tiempo. De esta forma se lograría una investigación de mayor calidad" (I357, 1:361).

El alumnado apunta a que el tipo de trabajos que evitarían tentaciones de plagio son los innovadores y de investigación "Disponiendo de tiempo para innovar, todo lo que nos piden y exigen en un trabajo en clase, está escrito, está hecho. Nos piden pocos trabajos de investigación" (I143, 1:147); motivadores "Establecer más motivación a la hora de elegir el tema de un proyecto. Muchas veces los temas son aburridos y conlleva a no sentir interés ni motivación al realizar el proyecto, produciéndose el plagio" (I237, 1:242); con utilidad: "Proponiendo temas que motiven, sean interesantes y que realmente le sirvan como formación académica" (I11, 1:11); personales y prácticos "Mandando trabajos más específicos donde no haya tanta información y que tengan un contenido más práctico que teórico" (I9, 1:9) y creativos "Fomentando la creatividad y expresión de los alumnos" (I238, 1:243).

Se sugieren trabajos donde se busque la calidad y no tanto la cantidad "Dando trabajos que generen más interés en el alumno y que le haga explotar sus conocimientos, es mejor mandar un trabajo bueno y no muchos mediocres ya que harán que el alumno pierda interés en hacerlos correctamente" (I165, 1:169), "Procurar la "calidad" de los trabajos (en 
lugar de cantidad) sería un método que los alumnos trabajen más, se esfuercen por el resultado" (I145, 1:149).

En el segundo nivel de seguimiento, se señala que la implicación docente es un factor fundamental para llevar un control progresivo de los avances académicos "Llevando a cabo una evaluación y seguimiento más continuo de los trabajos, programando así más tutorías, teniendo un trato más personal con los alumnos, sobre todo en trabajos importantes" (I69, 1:69). Para ello, se propone lo siguiente:

Creo que con un seguimiento semanal o quincenal por parte de los profesores en trabajos extensos les facilitaría la detención de textos o trabajos plagiados. En trabajos de menor extensión, utilizar los softwares destinados para ello creo que facilitaría la detención de los mismos (I148, 1:152).

Finalmente, con respecto al nivel de control, centrándose en el momento del proceso de enseñanza en el que se comprueba la validez de los trabajos, se establecen diferentes medidas:

De tres maneras diferentes o conjuntas se pueden usar para entrar el plagio: Puntuar, otorgar méritos al uso de referencias bibliográficas y al uso correcto de los sistemas de referenciado. Usar herramientas digitales de entrega que cotejen los trabajos con diferentes bases de datos (por ejemplo, Turnitin). Mejor formación del alumnado en el uso de los sistemas de referenciado (I618, 1:620).

Así, se propone que el proceso de citación y de elaboración de referencias bibliográficas se establezca como requisito obligatorio, criterio además que podría ser valorado positivamente si la labor se realiza correctamente "Poniendo como condición indispensable para la evaluación, citar. Así los profesores podrían comprobar si hay plagio o no" (I17, 1:17).

Otra cuestión relacionada es que la anterior actividad no se limite a ser calificada, sino que es determinante ofrecer el correspondiente feedback al alumnado:

Desde mi punto de vista considero que el profesorado debería involucrarse más con los trabajos que ellos mismos mandan, ya que de diez asignaturas que he cursado, me han mandado realizar trabajos en seis de ellas y solo me han explicado las calificaciones por el trabajo realizado en uno, ya que en el resto solo contaba el haberlas entregado (I158, 1:162).

Finalmente, para identificar trabajos con plagio se consideran como herramientas clave los programas de detección de plagio digitales. Así mismo, se hace hincapié en la posibilidad de disponer de aplicaciones web que ayuden en esta labor "Haciendo una formación adecuada no solo en cómo citar, sino también herramientas que son de utilidad para ello, a las que recurrir en caso, 
por ejemplo, de tener sobrecarga de trabajos o muchas fuentes" (I430, 1:436), "Una herramienta que haga las referencias automáticamente" (I632, 1:636) o

Mediante la tecnología blockchain (o cadena de bloques), que permitiría dejar circunstancia, de manera cuasi-inmutable, de la autoría y propiedad intelectual de cualquier documento, de manera inmediata, a nivel global y sin necesidad de instituciones intermediarias, unas con el consenso a nivel global de todos los usuarios integrantes de la cadena (I742, 1:747).

Figura 4

Categoría y códigos de medidas de supervisión. Los rectángulos denotan las categorías y códigos de análisis. Cada rectángulo recoge información que responde a la siguiente leyenda: $E=$ frecuencia y $D=$ densidad (número de códigos con los que está vinculado)

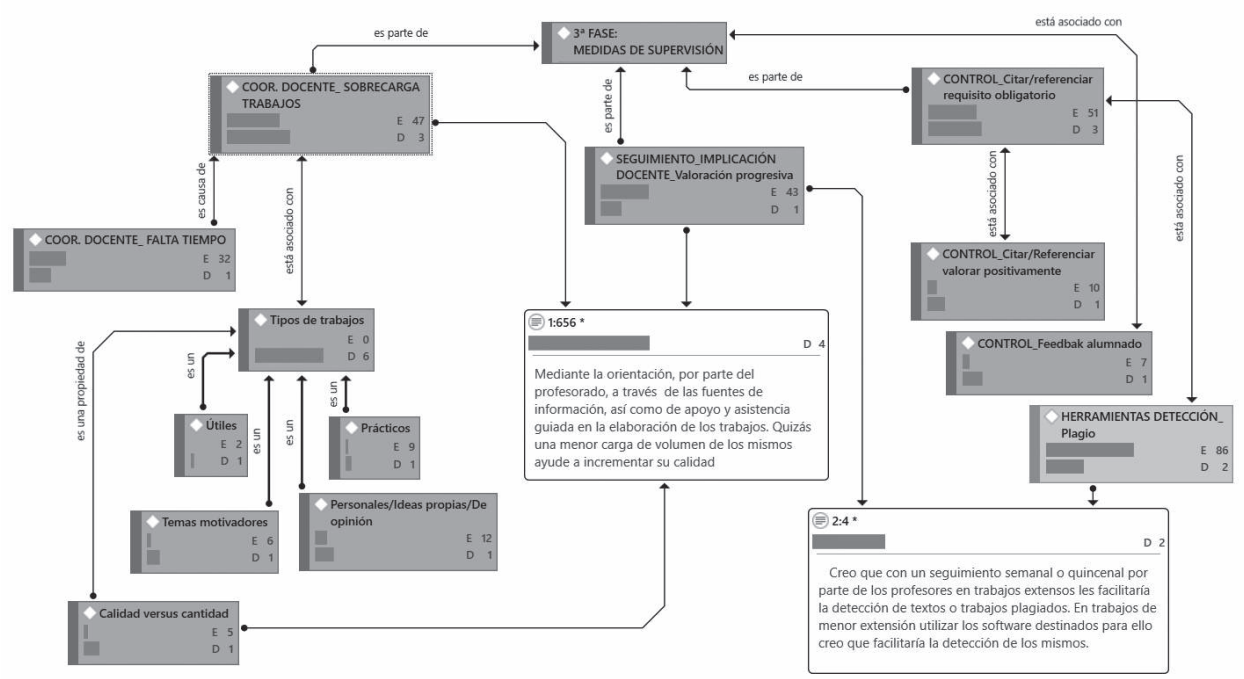

\section{Cuarta fase: medidas sancionadoras}

Una vez detectadas las infracciones con las herramientas digitales, la última fase, como se aprecia en la figura 5, consistiría en aplicar las medidas correctivas ante las acciones de plagio cometidas "Se puede acabar con el plagio utilizando programas de detección de fragmentos copiados y pegados y aplicando duras sanciones a los responsables" (I21, 1:21) o

Exponer ante todos los alumnos/as las directrices y medidas que puede tomar la universidad en caso de plagio, así como prestar más atención a los trabajos presentados por los alumnos verificando que no sean plagio, y en caso de serlo, sancionando debidamente (I161, 1:165).

El alumnado apunta varios tipos de sanciones: la pérdida del derecho a la evaluación continua o a la evaluación final, el suspenso en la parte práctica 
de la materia o en todo el trabajo, el pago de nueva matrícula e incluso la expulsión del alumnado, de acuerdo con las siguientes afirmaciones "Las correcciones de los profesores de los trabajos deben ser más profundas, así como las sanciones deben ser mayores, reflejándose en su nota o bien en la posibilidad de presentarse a los diferentes exámenes" (I53, 1:53), "Imponer sanciones más severas al que sea descubierto en cualquier actividad de este tipo. Como mínimo suspenso automático en la parte práctica de la asignatura" (I542, 1:543), "Creo que se puede evitar el plagio (copiar sin citar) amenazando con expulsar" (I603, 1:602) o

Los profesores tienen que dar las instrucciones necesarias para dar un guion y que sepamos lo que tenemos qué hacer. Sanciones de manera que nos influya en nuestras notas finales y así le cojamos miedo al copia y pega. También es importante que pierdas el derecho de evaluación y tengas que pagar otra matrícula, con dinero por medio siempre tienes más respeto (I170, 1:174).

Figura 5

Categoría y códigos de medidas sancionadoras. Los rectángulos denotan las categorías y códigos de análisis. Cada rectángulo recoge información que responde a la siguiente leyenda: E=frecuencia y $D=$ densidad (número de códigos con los que está vinculado)

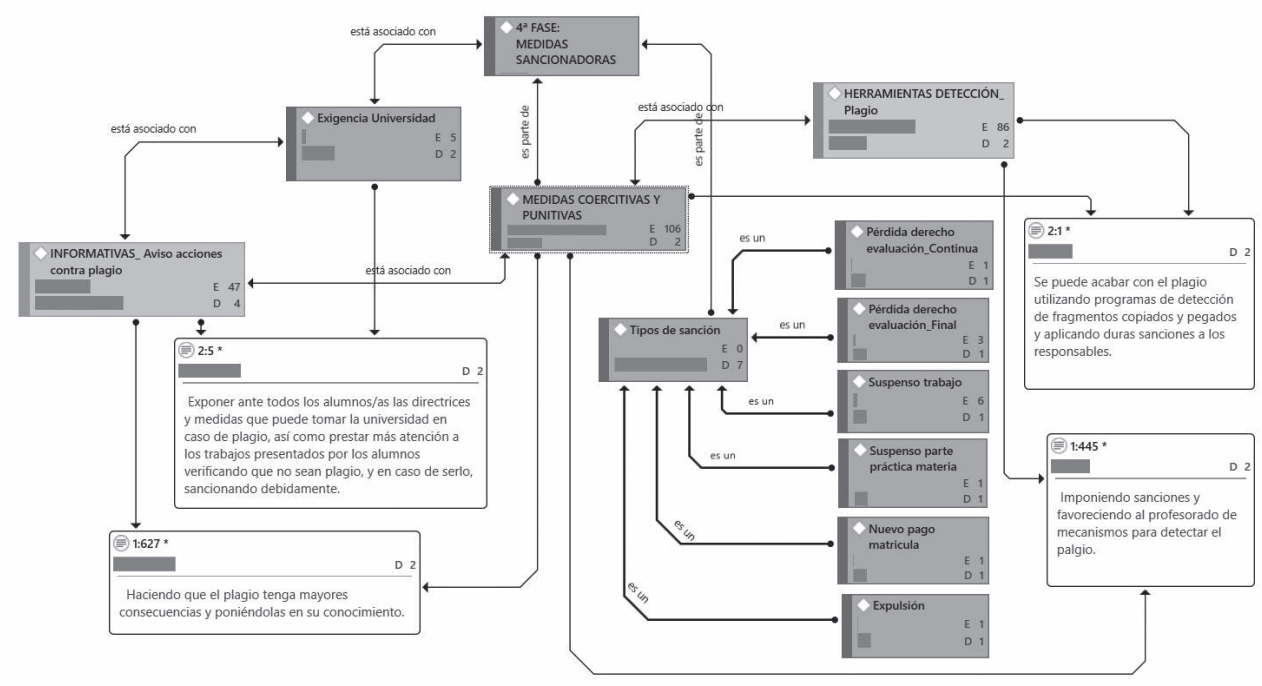

No obstante, también existen algunos casos en los que se considera que es algo inevitable de difícil solución:

Creo que el plagio no lo podemos evitar, simplemente hay quien trabaja y hay otros que prefieren copiar y engañarse a sí mismos porque todos sabemos lo que es copiado y lo que no. Se debe vigilar ese aspecto mucho más para reducirlo. Pero nunca desaparecerá (I560, 1:560) o 
Es algo casi imposible de evitar. Quizás mediante alguna página web que vea los plagios se podría, pero en los trabajos en papel es muy difícil. Además, el gran fallo es que la gran mayoría de los profesores no leen los trabajos que piden, por lo que el alumnado es comprensible que lo haga (I102, 1:105).

\section{DISCUSIÓN Y CONCLUSIONES}

La descripción que realiza el alumnado universitario acerca de las medidas para evitar el plagio ha de servir para diseñar y poner en marcha formas efectivas de atacarlo. El análisis de los resultados da lugar a conclusiones importantes a tener en cuenta, da respuesta a interrogantes efectuados en estudios previos y facilita asimismo nuevas posibilidades de investigación y de intervención. Así, se ha podido establecer un proceso de actuación docente, propuesto por el alumnado, que abarca las diferentes fases del proceso de elaboración de sus trabajos académicos y de su proceso de enseñanza-aprendizaje.

Las mejoras sugeridas se centran en medidas informativas justo en el momento de acceder a los estudios universitarios ya que, en la línea de lo manifestado por Miranda (2013) el alumnado admite que las acciones de comisión de plagio se producen normalmente por desconocimiento pero ya provienen de etapas educativas previas; en este sentido, se reclaman actuaciones dirigidas a informarles de la relevancia de no cometer plagio, sobre cómo acceder a fuentes y recursos de información y sobre cómo citar, pero también sobre la normativa que lo regula y las consecuencias que pueden acarrearles no hacerlo correctamente. Con respecto a estas medidas, el alumnado señala una doble vertiente de dicha información: a través de la propia universidad (CebriánRobles et al., 2018; Ramírez, 2017) pero también a través del personal docente. En este sentido, la universidad, como indican del Río y del Río (2020) ha de tener la responsabilidad de formar tanto al personal académico como al estudiantado "para que tengan conciencia y conocimiento de las bases éticas que sustentan las diversas comunicaciones científicas” (p. 66).

En segundo lugar, el estudiantado se centra en medidas de carácter formativo interrelacionando la importancia que tiene formar en el proceso de citación con la labor docente en el proceso de elaboración de sus trabajos académicos; pero también por parte de la universidad en relación con la reclamación de normativas del proceso de citación de carácter unificado. En este sentido, se sugiere entregar resúmenes de los manuales de citación, impartir clases de carácter teórico y práctico o dedicar una parte del tiempo de explicación de los trabajos a hablar sobre ello. Nuevamente se señala que esperar al último curso para realizar estas acciones no es positivo y se hace hincapié, en este caso, en el personal docente como referente en línea con la postura pedagógica que establecen Ordóñez et al. (2006) y Ochoa y Cueva (2016), que ha de incluir las referencias consultadas en los apuntes que les proporciona o tenerlas presentes en el momento de revisar sus trabajos académicos. En cuanto a las instrucciones 
que facilita el profesorado con respecto a los trabajos solicitados, establecen además medidas centradas en la necesidad de una mayor orientación y dirección por parte del profesorado, señalando sentirse "abandonados" durante el proceso de elaboración y se solicitan acciones destinadas a incrementar factores de carácter personal relacionados con la concienciación por parte del alumnado, su interés y su responsabilidad, valorando la importancia que tendrá en sus trabajos que el proceso de citación sea correcto (Escalante e Ibarra, 2013). Llergo y Alvear (2020) se decantan más por este tipo de medidas formativas que por "medidas anti plagio" destacando, en primer grado de importancia, la "formación en honradez intelectual" (p. 90).

En tercer lugar, la supervisión de los trabajos académicos, según el alumnado, recae en el profesorado que ha de coordinarse con el resto de docentes a la hora de solicitarlos, teniendo en cuenta el número de trabajos requeridos y el tiempo asignado para efectuarlos. Se demanda que el tipo de trabajos solicitados sea de calidad, motivadores, prácticos, de utilidad para su futuro profesional y en los que tengan que desarrollar y demostrar su esfuerzo personal (Mejía y Ordóñez, 2004). Durante su desarrollo se exige también un seguimiento continuado, que, como señala Salazar (2020), podría efectuarse a través de correo electrónico o mediante la entrega de los trabajos académicos en las aulas virtuales. De este modo se conseguiría una evaluación personalizada a través de tutorías y un control en relación al plagio, que ha de ser previamente establecido en los criterios de evaluación a través de un proceso de coevaluación entre alumnado y profesorado con la correspondiente retroalimentación (Cebrián-Robles et al., 2018; Zarfsaz y Ahmadi, 2017), determinando la importancia de la evaluación en la "delimitación del saber ser educativo" (Jiménez, 2019, p. 185) y empleando para ello herramientas de detección a las que, en su opinión, han de tener acceso, lo cual permitiría revisar la originalidad de dichos trabajos, así como su adecuación a las normas de publicación de trabajos escritos (Salazar, 2020). También es interesante el estudio efectuado por Soto et al. (2020) con alumnado de doctorado a través de la aplicación iThenticate mediante la cual se analiza la copia, el parafraseo incorrecto y la ausencia de citas, consiguiéndose, mediante un proceso de retroalimentación al estudiantado, rebajar el índice de similitud en los trabajos académicos.

La evitación del plagio ha de pasar por poner los medios que permitan adquirir conciencia de que en las universidades existen reglas morales que no se deben obviar, previniendo, enseñando, pero también sancionando; destacan la variedad de medidas sancionadoras propuestas por el alumnado, tanto de carácter punitivo como coercitivo, en línea con lo establecido por Miranda (2013) y al contrario de lo indicado por Cebrián-Robles et al. (2018). Sería interesante conocer si los tipos de sanciones que proponen variarían en función del tipo de falta cometida o su nivel de gravedad, tal y como han estudiado Sureda-Negre et al. (2020).

Este estudio facilita nuevas posibilidades de intervención que se centren en diseñar y poner en marcha guías de actuación, tomar decisiones en cuanto al 
momento de explicar al alumnado las normativas y las sanciones determinadas por cada universidad o discutir en futuros estudios la responsabilidad docente como elemento fundamental en la concienciación del alumnado, a través de procesos de orientación académica personalizados así como la responsabilidad del alumnado en su propio proceso de enseñanza-aprendizaje. También sería interesante realizar en próximos estudios una triangulación de los datos con el fin de presentar resultados independientes por cada uno de los grupos informantes. 


\section{REFERENCIAS BIBLIOGRÁFICAS}

Akbulut, Y., Sendag, S., Birinci, G., Kiliçer, K., Sahin, M., \& Odabasi, H.F. (2008). Exploring the types and reasons of interet-triggered academic dishonesty among Turkish undergraduate students: development of internet-triggered academic dishonesty scale (ITADS). Computers \& Education 51(1), 463-473. https://doi. org/10.1016/j.compedu.2007.06.003

Ardila, E.E., \& Rueda, J.F. (2013). La saturación teórica en la teoría fundamentada: su delimitación en el análisis de trayectorias de vida de víctimas del desplazamiento forzado en Colombia. Revista Colombiana de Sociología, 36(2), 93-114. https:// revistas.unal.edu.co/index.php/recs/ article/view/41641

Barry, C.A., Britten, N., Barber, N., Bradley, C., \& Stevenson, F. (1999). Using reflexivity to optimize teamwork in qualitative research. Qualitative Health Research, 9(1), 26-44. https://doi. org/10.1177\%2F104973299129121677

Caldevilla, D. (2010). Internet como fuente de información para el alumnado universitario. Cuadernos de Documentación Multimedia, 21, 141-157. https://revistas.ucm. es/index.php/CDMU/article/view/ CDMU1010110141A

Cebrián-Robles, V., Raposo-Rivas, M., \& Sarmiento-Campos, J.A. (2016). ¿Ética o prácticas deshonestas? El plagio en las titulaciones de Educación. Revista de Educación, 374, 161186. https://doi.org/10.4438/1988592X-RE-2016-374-330

Cebrián-Robles, V., Raposo-Rivas, M., Cebrián-de-la-Serna, M., \& SarmientoCampos, J. A. (2018). Percepción sobre el plagio académico de estudiantes universitarios españoles. Educación $X X 1,21(2)$, 105-129. https://doi. org/10.5944/educXX1.20062

Creswell, J.W. (1998). Qualitative inquiry and research design. Choosing among five traditions. SAGE Publications.

Del Río, J.H., \& del Río, D.C. (2020). Ética y conductas inapropiadas en la práctica de la investigación. Medicina y Ética, 31(1), 49-69. https://doi. org/10.36105/mye.2020v31n1.02

Dias, P.C., Bastos, A.S., Gandra, M., \& Díaz-Pérez, J. (2013). Genius, ¿plagio o creatividad? Aportes para una discusión sobre las prácticas pedagógicas. Bordón, 65(3), 9-23. https://recyt.fecyt.es/index.php/ BORDON/article/view/23154/11590

Escalante, A.E., \& Ibarra, L.M. (2013). Dilemas que enfrenta el estudiantado de postgrado en su formación ética como investigador. Edetania. Estudios Y Propuestas Socioeducativas, 43, 6781. https://revistas.ucv.es/index.php/ Edetania/article/view/216

Flick, U. (2012). Introducción a la investigación cualitativa. Ediciones Morata.

Flick, U. (2014). La gestión de la calidad en investigación cualitativa. Ediciones Morata.

Froment, F., Bohórquez, R., \& GarcíaGonzález, A.J. (2020). Credibilidad docente: una revisión de la litaratura. TERI, 32(1), 23-54. https://doi. org/10.14201/teri.20313

García-Peñalvo, F.J. (2017). Mitos y realidades del acceso abierto. Education in the Knowledge Society, 18(1), 7-20. https://doi.org/10.14201/ eks2017181720 
Hwang, S. (2007). Utilizing qualitative data analysis software: A review of ATLAS.ti. Social Science Computer Review, 26(4), 519-527. https://doi. org/10.1177/0894439307312485

Jiménez, J.A. (2019). Aproximaciones epistemológicas de la evaluación educativa: entre el deber ser y lo relativo. Foro de Educación, 17(27), 185-202. https://doi.org/10.14516/ fde. 636

Kuntz, J.R.C., \& Buttler, C. (2014). Exploring individual and contextual antecedents of attitudes toward the acceptability of cheating and plagiarism. Ethics \& Behavior, 24(6), 478-494. https://doi.org/10.1080/1050 8422.2014 .908380

Llergo, F., \& Alvear, M.P. (2020). Frente al plagio: buenas prácticas, alegría y ecología en el aula. Revista Panamericana de Pedagogía. 29, 89109.

Mejía, J.F., \& Ordóñez, C.L. (2004). El fraude académico en la Universidad de los Andes ¿qué, qué tanto y por qué? Revista de Estudios Sociales, 18, 13-25. https://doi.org/10.7440/res18.2004.01

Mendoza, A. (2019). Los saberes de la era digital. Nuevas tecnologías para la práctica humanística: teoría del aprendizaje, bibliotecas digitales y arte terapia. Revista Española de Educación Comparada, 35, 227-229.

Miles, M., \& Huberman, M. (1994). Qualitative Data Analysis. An expanded sourcebook. SAGE publications.

Miles, Huberman, M., \& Saldaña, J. (2014). Qualitative Data Analysis. A Methods Sourcebook. SAGE Publications.

Miranda, A. (2013). Plagio y ética de la investigación científica. Revista Chilena de Derecho, 40(2), 711-
726. https://doi.org/10.4067/S071834372013000200016

Morey-López, M., Sureda-Negre, J., Oliver-Trobat, M., \& Comas-Forgas, R.L. (2013). Plagio y rendimiento académico entre el alumnado de Educación Secundaria Obligatoria. Estudios sobre Educación, 24, 225-244. https://hdl.handle.net/10171/29571

Muñoz-Cantero, J.M., Rebollo-Quintela, N., Mosteiro-García, M.J., \& OcampoGómez, C.I. (2019). Validación del cuestionario de atribuciones para la detección de coincidencias en trabajos académicos. Revista Electrónica de Investgiación y Evaluación Educativa, 25(1). https://doi.org/10.7203/ relieve.25.1.13599

Ochoa, L., \& Cueva, A. (2016). Percepciones de estudiantes acerca del plagio: datos cualitativos. Revista Encuentros, 14(2), 25-41. https://doi. org/10.15665/re.v14i2.822

Ordóñez, C.L., Mejía, J.F., \& Castellanos, S. (2006). Percepciones estudiantiles sobre el fraude académico: hallazgos y reflexiones pedagógicas. Revista de Estudios Sociales, 23, 37-44.

Ponte, E.J. (2020). Guía metodológica para el desarrollo de trabajos científicos en el nivel de conocimientos de investigación de estudiantes universitarios. [Tesis doctoral, Universidad César Vallejo]. http://repositorio.ucv.edu.pe/ handle/20.500.12692/41950

Pujadas, J. (2000). El método biográfico y los géneros de la memoria. Revista de Antropología Social, 9, 127-158. https:// revistas.ucm.es/index.php/RASO/ article/view/RASO0000110127A

Ramírez, A.C. (2017). El plagio académico. Experiencias y algunas ideas para desalentarlo de manera más efectiva. Ciencia Nicoalita, 70, 
7-22. https://www.cic.cn.umich.mx/ cn/article/view/355

Real Decreto 1027/2011, de 15 de julio, por el que se establece el Marco Español de Cualificaciones para la Educación Superior. Boletín Oficial del Estado, núm. 185, de 3 de agosto de 2011.

Rodríguez, J., Artiles, J., Guerra, M. \& Mena, J.L. (2020). Aprendizaje del estudiante universitario: un estudio de caso. Educar, 56(1), 201-217. https:// doi.org/10.5565/rev/educar.1087

Salazar, C. (2020). Incorporación de las TIC en la enseñanza y el aprendizaje de la lectura y la escritura en la universidad. En I.A. Molina, J.C. Morales, \& S.A. Rodríguez (Eds.), Importancia de las TIC en los procesos de enseñanza-aprendizaje. Estudios en la Educación Media y Superior (pp. 163-177). Universidad Sergio Arboleda.

Sandín, M.P. (2000). Criterios de validez en la investigación cualitativa: de la objetividad a la solidaridad. Revista de Investigación Educativa, 18(1), 223242. https://revistas.um.es/rie/article/ view/121561/114241

Sarmiento-Campos, J.A., OcampoGómez, C.I., Barreira-Arias, A.J., Castro-Pais, M.D., \& Rodríguez, P. (2019). El plagio en la Educación Superior: estudio estadístico textual de las opiniones del estudiantado. En M. Peralbo, A. Risso, A. Barca, B. Duarte,
L. Almeida, \& J.C. Brenlla (Eds.), Actas del XV Congreso Internacional GallegoPortugués de Psicopedagogía (pp. 3569-3584). Universidade da Coruña, Servicio de Publicaciones. https://doi. org/10.17979/spudc.9788497497268

Soto, J.L., Hernández, E., Zamudio, J.C., \& Torres, C.A. (2020). Valoración del plagio en ensayos elaborados por estudiantes de un doctorado en línea. Praxis Educativa REDIE., 21(11), 133135.

Sureda-Negre, J., Cerdà-Navarro, A., Calvo-Sastre, A. \& Comas-Forgas, R. (2020). Las conductas fraudulentas del alumnado universitario español en las evaluaciones: valoración de su gravedad y propuestas de sanciones a partir de un panel de expertos. Revista de Investigación Educativa, 38(1), 201209. https://doi.org/10.6018/rie.358781

Tayan, B. M. (2017). Academic misconduct: an investigation into male students' perceptions experiences \& attitudes towards cheating and plagiarism in a Middle Eastern University context. Journal of Education and Learning, 6(1), 158-166. https://doi.org/10.5539/jel.v6n1p158

Zarfsaz, E., \& Ahmadi, R. (2017). Investigating some main causes and reasons of writing plagiarism in a EFL context. International Journal of Applied Linguistics \& English Literature, 6(5), 214-223. https://doi. org/10.7575/aiac.ijalel.v.6n.5p.214 


\section{PERFIL ACADÉMICO Y PROFESIONAL DE LOS AUTORES}

Jesús Miguel Muñoz Cantero. ORCID: https://orcid.org/0000-0001-5502-1771

Catedrático de universidad. Coordinador de los Grupos GIACE (Grupo de Investigación en Evaluación y Calidad Educativa) y GITIAES (Grupo de Innovación en Tutorización y Apoyo al Estudiantado). Líneas de investigación: evaluación y calidad educativa. Líneas de innovación: plagio académico. E-mail: jesus.miguel.munoz@udc.es

Eva María Espiñeira Bellón. ORCID: https://orcid.org/0000-0001-7522-9406

Profesora contratada doctora. Miembro de los grupos Evaluación y Calidad en Educación (GIACE) y Tutorización y Apoyo al Estudiantado (GITIAES). Líneas de investigación: atención a la diversidad e inclusión socioeducativa. Líneas de innovación: plagio académico.

E-mail: eva.espineira@udc.es

María Cristina Pérez Crego. ORCID: https://orcid.org/0000-0001-5937-0047

Profesora interina de sustitución. Miembro de los grupos Evaluación y Calidad en Educación (GIACE) y Tutorización y Apoyo al Estudiantado (GITIAES). Líneas de investigación: orientación educativa, método biográfico-narrativo, Educación para el Desarrollo. Líneas de innovación: plagio académico. E-mail: cristina.pcrego@udc.es

Fecha Recepción del Artículo: 22. Septiembre. 2020

Fecha Modificación del Artículo: 07. Febrero. 2021

Fecha Aceptación del Artículo: 09. Febrero. 2021

Fecha Revisión para Publicación: 25. Febrero. 2021 
